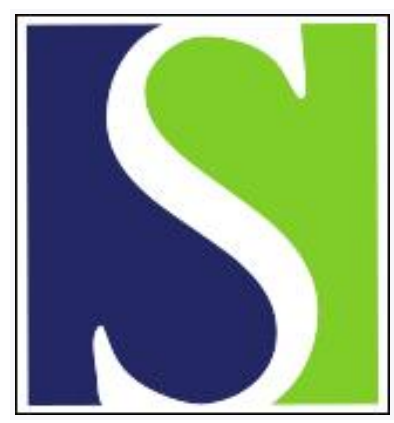

Scand J Work Environ Health 1997;23(6):403-413

https://doi.org/10.5271/sjweh.262

Issue date: Dec 1997

\title{
Predictors of disability retirement
}

by Krause N, Lynch J, Kaplan GA, Cohen RD, Goldberg DF, Salonen JT

The following articles refer to this text: 2003;29(3):167-169;

2003;29(3):171-188; 2006;32(6):431-442; 2006;32(6):502-514;

2008;34(6):479-482; 2008;34(6):483-486; 2009;35(1):56-64;

2011;37(6):455-463; 2011;37(6):464-472; 2011;37(6):451-453;

2012;38(5):391-392; 2013;39(5):468-476; 2013;39(6):609-617;

2014;40(5):483-492; 2016;42(2):135-143

Key terms: disability; disability retirement; occupational health; prospective study; psychological strain; retirement; risk factor; stress; unemployment; workload

This article in PubMed: www.ncbi.nlm.nih.gov/pubmed/9476803 


\title{
Predictors of disability retirement
}

\author{
by Niklas Krause, MD, ${ }^{1}$ John Lynch, PhD, ${ }^{2}$ George A Kaplan, PhD, ${ }^{2}$ Richard D Cohen, MA, ${ }^{1}$ \\ Debbie E Goldberg, MS, ${ }^{1} J u k k a$ T Salonen, $M D^{3}$
}

\author{
Krause N, Lynch J, Kaplan GA, Cohen RD, Goldberg DF, Salonen JT. Predictors of disability retirement. Scand \\ $J$ Work Environ Health 1997;23(6):403-13.
}

\begin{abstract}
Objectives Disability retirement may increase as the work force ages, but there is little information on factors associated with retirement because of disability. This is the first prospective population-based study of predictors of disability retirement including information on workplace, socioeconomic, behavioral, and health-related factors.

Methods The subjects were 1038 Finnish men who were enrolled in the Kuopio Ischemic Heart Disease Risk Factor Study, who were $42,48,54$, or 60 years of age at the beginning of the study, and who participated in a 4-year follow-up medical examination.

Results Various job characteristcs predicted disability retirement. Heavy work, work in uncomfortable positions, long workhours, noise at work, physical job strain, musculoskeletal strain, repetitive or continuous muscle strain, mental job strain, and job dissatisfaction were all significantly associated with the incidence of disability retirement. The ability to communicate with fellow workers and social support from supervisors tended to reduce the risk of disability retirement. The relationships persisted after control for socioeconomic factors, prevalent disease, and health behavior, which were also associated with disability retirement.

Conclusions The strong associations found between workplace factors and the incidence of disability retirement link the problem of disability retirement to the problem of poor work conditions.
\end{abstract}

Key terms disability, occupational health, prospective studies, retirement, risk factors, stress, psychological, unemployment, work load.

Nearly one-third of the world's workers are over 45 years of age, and this proportion is increasing rapidly. In Finland, this proportion is projected to increase to over $41 \%$ during the next 30 years (1). An aging work force may be more likely to have increased rates of early retirement due to disability. Little information is available about which aspects of the work environment or of the worker's life are importantly related to disability retirement. Most of the epidemiologic work in this area has focused on factors associated with early or old-age retirement $(2$, 3 ) or on postretirement health effects $(4,5)$ rather than on disability retirement per se.

However, some recent studies have indicated a possible role of workplace factors in premature retirement (611). Even though information on the work environment is limited and the evidence is based either on a cross- sectional analysis or is restricted to nonrepresentative company-based samples, these studies highlight the fact that workplace conditions may be important in disability retirement. From a public health perspective, factors related to the nature of work are especially important since many of them may be amenable to intervention.

This study is the first prospective investigation of risk factors for disability retirement in a population-based sample which includes measures of workplace conditions, socioeconomic factors, health status, and behavioral factors. The objectives of this study were to investigate whether physical and organizational work conditions and perceptions of job strain, job dissatisfaction, and social support predict disability retirement independent of socioeconomic factors, prevalent disease, and health behavior.

1 Human Population Laboratory, Public Health Institute, Berkeley, California, United States.

2 Department of Epidemiology, School of Public Health, University of Michigan, Ann Arbor, Michigan, United States.

3 Research Institute of Public Health, University of Kuopio, Kuopio, Finland.

Reprint orders to: Dr Niklas Krause, School of Public Health, Division of Epidemiology, 140 Warren Hall, University of California, Berkeley, CA 94720_7360. [E-mail: nkrause@uclink4.berkeley.edu] 


\section{Subjects and methods}

\section{Subjects}

The subjects were participants in the Kuopio Ischemic Heart Disease Risk Factor Study, which was designed to investigate risk factors for ischemic heart disease, carotid atherosclerosis, and other related outcomes in a population-based $30 \%$ random sample of Eastern Finnish men (12). Details of the study sample have been published elsewhere $(13,14)$. Base-line examinations were conducted between March 1984 and December 1989 on 2682 men (participation rate $82.9 \%$ ) who resided in the city of Kuopio or its surrounding rural communities. Follow-up examinations were conducted on an age-stratified sample of men who underwent ultrasonic scans at the beginning of the study. On the average, the participants were followed for 4.2 (range $3.8-5.2$ ) years. Of the 1229 participants who were eligible for the follow-up examinations, 52 had died, were suffering severe illness, or had migrated away from the area. Of the remainder, 139 could not be contacted or refused to participate. The participation rate calculated for the potentially available subjects at follow-up was $88.2 \%$. The number of participants in the 42-, 48-, 54-, and 60-year-old age cohorts was $236,247,284$, and 271, respectively. Complete information on retirement status, demographic, economic, disease, and health behavior variables was available for 968 participants. All the analyses were restricted to these 968 participants to ensure comparability of the models which adjusted for different sets of covariates. The sample size for the models analyzing individual workplace factors varied from 648 to 968 due to missing values for some job variables. During the 4-year follow-up, 67 new cases of disability retirement were observed among the 968 participants $(14.5 \%)$ ). At the beginning of the study, $81 \%$ of these persons had been working full-time and $6 \%$ part-time, whereas $7 \%$ had been unemployed or temporarily layed off, and 6\% were not working for other reasons.

\section{Ascertainment of disability retirement}

Retirement status was ascertained by questionnaire at at the beginning of the study and at the time of the followup examination. Response options included disability retired, partly retired, early retired, retired on pension (without disability), temporarily laid off, unemployed, and for some other reason not working. Only the men who were disability retired at the time of the follow-up but were not retired in any form at the beginning of the study were included as incident cases.

\section{Workplace factors}

Over 100 questions tapped the physical and organizational characteristics of the work environment and the psychological and physical demands experienced by the working men. Some variables which assessed the same underlying work domain were combined to form indices. Internal consistency was validated by calculating Cronbach's alpha coefficients for each index. (See the appendix.) Individual questionnaire items were modeled dichotomally, while the indices were modeled as the median split, tertiles, quartiles, or quintiles according to their distributions.

There were 2 broad categories of workplace factors: (i) variables describing physical and organizational work conditions and (ii) variables that, in part, reflect the person's appraisal of the stress or strain induced by work conditions. The first category included physical factors such as heavy physical work, repetitive straining of muscles, frequency of various work positions, exposure to noise, and organizational factors such as weekly workhours, shift work, piece-work, isolation at work, frequency of deadlines, and signs of overwork. Variables in the second category included ratings of physical strain, back strain, neck strain, strain in various parts of the extremities, cardiorespiratory strain, mental strain, mental strain from strenuous work, time pressure, tiredness after work, job dissatisfaction, and co-worker and supervisor support.

\section{Covariates}

Various covariates were assessed, including socioeconomic factors, prevalent disease, and health behavior.

Socioeconomic factors. Socioeconomic factors were assessed by questionnaire at the beginning of the study and included age, education, occupation, marital status, number and age of dependents, place of residence, income, unemployment during the past 5 years, number of family members working, number of family members unemployed, and whether the respondent's wife stayed at home (if married).

Prevalent diseases. Disease status was determined as part of a 2-day examination at the beginning of the study, including medical history, physical examination, a battery of laboratory tests, electrocardiograms, and measurement of respiratory gas exchange during symptomlimited maximum exercise tolerance tests on a bicycle ergometer, among others. Prevalent diseases were grouped into the following categories: serious injury in the past, osteoarthritis, rheumatoid arthritis, back problems, headaches, digestive tract disease, urinary tract disease, respiratory disease, symptomatic coronary heart disease [defined by medical history, the Rose Angina Questionnaire (15), electrocardiographic changes or the use of anti-angina medication], and asymptomatic coronary heart disease (indicated by ischemia during maximal exercise tolerance tests on a bicycle ergometer in the 
absence of prior diagnosis or pain), hypertension, stroke, diabetes, obesity, neurological disorders, mental illness, and depression [MMPI T-score >70 (16)]. Obesity was defined as body mass index greater than $30 \mathrm{~kg} / \mathrm{m}^{2}$. In addition, cardiorespiratory fitness $\left(\dot{\mathrm{VO}}_{2} \max \right)$ and selfperceived health were included as general health indicators. Cardiorespiratory fitness was assessed by respiratory gas exchange during a maximal, symptom-limited exercise tolerance test.

Health behavior. Alcohol consumption during a 4-day period and during the last 12 months was assessed by dietary recall and by a self-administered questionnaire (17). Smoking was measured by questionnaire and classified for this analysis as "never smoker", "former smoker", and "current smoker". Physical activity was assessed at the beginning of the study from a 12-month leisuretime history. The present analyses use total duration (frequency multiplied by the duration of sessions of leisuretime physical activity), which has been shown to be predictive of myocardial infarction in this population (19).

\section{Statistical analysis}

For each variable the association with incidence of disability retirement was examined in age-adjusted logistic regression models. For each workplace variable, additional analyses were performed by incrementally adding conceptually related groups of variables to each model. First, economic variables that concerned possible incentives for retirement were added to each model, then prevalent diseases were added, followed by variables measuring health behavior. All analyses were performed using the LOGISTIC procedure in SAS Version 6.09 on a Sun SPARC Station 20 (19).

\section{Results}

\section{Socioeconomic factors}

Table 1 presents the age-adjusted associations between socioeconomic factors and the incidence of disability retirement. The 54-year-old men were at the highest risk for disability retirement when compared with the men 42 years of age at the beginning of the study (odds ratio (OR) $3.59,95 \%$ confidence interval (95\% CI) $1.74-$ 7.39). Education showed an inversely graded risk. Marked differences were found for occupational groups analyzed by economic sector; blue-collar workers $(\mathrm{N}=388)$, self-employed farmers $(\mathrm{N}=88)$, and self-employed professionals or entrepreneurs $(\mathrm{N}=102)$ had odds ratios of $2.14(95 \%$ CI $1.11-4.11), 3.17$ (95\% CI 1.36$7.38)$, and 4.67 (2.15-10.17), respectively, when compared with white-collar workers $(\mathrm{N}=339)$. Farm and forestry workers $(\mathrm{N}=33)$ were excluded from this anal- ysis because there were too few men and no incident cases of disability retirement in this group. Unemployment for any period of time during the 5 years prior to the beginning of the study predicted disability retirement (OR 1.98, 95\% CI 1.07-3.63) with the highest risk for those still unemployed at the beginning of the study (OR $3.45,95 \% \mathrm{CI} 0.88-13.49$ ). An inverse association was

Table 1. Age-adjusted associations of socioeconomic factors with incidence of disability retirement.

\begin{tabular}{|c|c|c|c|}
\hline Variable & $\begin{array}{l}\text { Adjusted } \\
\text { odds ratio }\end{array}$ & $\begin{array}{l}95 \% \\
\text { confidence } \\
\text { interval }\end{array}$ & P-value \\
\hline \multicolumn{4}{|l|}{ Age } \\
\hline $\begin{array}{l}42 \text { years } \\
48 \text { years } \\
54 \text { years } \\
60 \text { years }\end{array}$ & $\begin{array}{l}1.00 \\
0.90 \\
3.59 \\
1.03\end{array}$ & $\begin{array}{l}0.36-2.26 \\
1.74-7.39 \\
0.43-2.48\end{array}$ & $\begin{array}{l}0.822 \\
0.001 \\
0.940\end{array}$ \\
\hline \multicolumn{4}{|l|}{ Marital status } \\
\hline $\begin{array}{l}\text { Married } \\
\text { Single } \\
\text { Divorced or widowed }\end{array}$ & $\begin{array}{l}1.00 \\
1.02 \\
1.94\end{array}$ & $\begin{array}{l}0.30-3.45 \\
0.86-4.37\end{array}$ & $\begin{array}{l}0.976 \\
0.109\end{array}$ \\
\hline \multicolumn{4}{|l|}{ Wife } \\
\hline $\begin{array}{l}\text { Not at home } \\
\text { At home }\end{array}$ & $\begin{array}{l}1.00 \\
1.16\end{array}$ & $0.65-2.09$ & 0.615 \\
\hline \multicolumn{4}{|l|}{ Residence } \\
\hline $\begin{array}{l}\text { Kuopio city } \\
\text { Town dwellers } \\
\text { Rural }\end{array}$ & $\begin{array}{l}1.00 \\
0.65 \\
1.38\end{array}$ & $\begin{array}{l}0.30-1.44 \\
0.79-2.40\end{array}$ & $\begin{array}{l}0.291 \\
0.255\end{array}$ \\
\hline \multicolumn{4}{|l|}{ Education } \\
\hline $\begin{array}{l}\text { High school or higher } \\
\text { Junior high } \\
\text { Primary } \\
\text { Less than primary school }\end{array}$ & $\begin{array}{l}1.00 \\
0.80 \\
1.40 \\
2.00\end{array}$ & $\begin{array}{l}0.29-2.21 \\
0.52-3.76 \\
0.62-6.43\end{array}$ & $\begin{array}{l}0.664 \\
0.502 \\
0.246\end{array}$ \\
\hline \multicolumn{4}{|l|}{ Occupation } \\
\hline $\begin{array}{l}\text { White collar } \\
\text { Blue collar } \\
\text { Self-employed farmer } \\
\text { Self-employed entrepreneur }\end{array}$ & $\begin{array}{l}1.00 \\
2.14 \\
3.17 \\
4.67\end{array}$ & $\begin{array}{l}1.11-4.11 \\
1.36-7.38 \\
2.15-10.17\end{array}$ & $\begin{array}{l}0.023 \\
0.008 \\
0.000\end{array}$ \\
\hline \multicolumn{4}{|l|}{ Income } \\
\hline $\begin{array}{l}\text { First quintile } \\
\text { Second quintile } \\
\text { Third quintile } \\
\text { Fourth quintile } \\
\text { Fifth quintile (lowest income) }\end{array}$ & $\begin{array}{l}1.00 \\
0.98 \\
1.10 \\
0.97 \\
1.20\end{array}$ & $\begin{array}{l}0.48-1.98 \\
0.52-2.34 \\
0.43-2.16 \\
0.51-2.84\end{array}$ & $\begin{array}{l}0.948 \\
0.808 \\
0.947 \\
0.680\end{array}$ \\
\hline \multicolumn{4}{|l|}{ Unemployment } \\
\hline $\begin{array}{l}\text { None } \\
\text { In past } 5 \text { years } \\
\text { Currently }\end{array}$ & $\begin{array}{l}1.00 \\
1.98 \\
3.45\end{array}$ & $\begin{array}{l}1.07-3.63 \\
0.88-13.49\end{array}$ & $\begin{array}{l}0.028 \\
0.076\end{array}$ \\
\hline \multicolumn{4}{|l|}{ Family members unemployed } \\
\hline $\begin{array}{l}\text { None } \\
\text { One } \\
\text { Two or more }\end{array}$ & $\begin{array}{l}1.00 \\
0.69 \\
0.22\end{array}$ & $\begin{array}{l}0.38-1.24 \\
0.06-0.78\end{array}$ & $\begin{array}{l}0.214 \\
0.019\end{array}$ \\
\hline \multicolumn{4}{|l|}{ Family members working } \\
\hline $\begin{array}{l}\text { None } \\
\text { One } \\
\text { Two } \\
\text { Three } \\
\text { Four or more }\end{array}$ & $\begin{array}{l}1.00 \\
3.42 \\
3.25 \\
4.51 \\
5.04\end{array}$ & $\begin{array}{l}0.98-11.99 \\
0.92-11.45 \\
1.12-18.16 \\
0.73-34.74\end{array}$ & $\begin{array}{l}0.054 \\
0.067 \\
0.034 \\
0.101\end{array}$ \\
\hline
\end{tabular}

a Age adjustment was performed for all the explanatory variables but age. The age-adjusted odds ratios are based on logistic regression models including indicator variables for the categories of one explanatory variable and four indicator variables for the age strata $42,48,54$, and 60 years.

- Thirty-three farm and forest workers were excluded from the analysis of occupation because no disability retirement occurred in this group; 18 participants were excluded because of unknown occupation. 
found for unemployment among family members. When 2 or more family members were unemployed at the beginning of the study, the risk for disability retirement was reduced by a factor of 4.6 (OR $0.22,95 \%$ CI 0.06

Table 2. Age-adjusted associations of prevalent disease, cardiorespiratory fitness, and self-perceived health with incidence of disability retirement. (MMPI = Minnesota Multiphasic Personality Inventory, $\mathrm{BMI}=$ body mass index, $\mathrm{CHD}=$ coronary heart disease, $\dot{V}_{2}$ max $=$ maximal oxygen consumption)

\begin{tabular}{|c|c|c|c|}
\hline Variable $^{a}$ & $\begin{array}{l}\text { Adjusted } \\
\text { odds ratio }\end{array}$ & $\begin{array}{c}95 \% \\
\text { confidence } \\
\text { interval }\end{array}$ & $P$-value \\
\hline Serious injury in the past & 2.73 & $1.45-5.15$ & 0.002 \\
\hline Osteoarthritis & 2.19 & $1.24-3.86$ & 0.007 \\
\hline Rheumatoid arthritis & 1.51 & $0.56-4.03$ & 0.413 \\
\hline Back problems & 1.60 & $0.97-2.67$ & 0.068 \\
\hline Migraine or headache & 2.09 & $1.12-3.89$ & 0.021 \\
\hline Depression (MMPI T > 70) & 1.52 & $0.91-2.54$ & 0.106 \\
\hline Mental illness & 0.49 & $0.12-2.08$ & 0.335 \\
\hline Obesity (BMI > 30) & 1.46 & $0.43-2.48$ & 0.189 \\
\hline Digestive tract disease & 0.70 & $0.36-1.39$ & 0.310 \\
\hline Urinary tract disease & 1.66 & $0.71-3.90$ & 0.241 \\
\hline Respiratory disease & 1.16 & $0.56-2.38$ & 0.692 \\
\hline Symptomatic CHD & 1.40 & $0.79-2.47$ & 0.251 \\
\hline Asymptomatic CHD & 0.35 & $0.13-0.99$ & 0.048 \\
\hline Hypertension & 1.06 & $0.63-1.80$ & 0.820 \\
\hline \multicolumn{4}{|l|}{$\begin{array}{l}\text { Cardiorespiratory fitness } \\
\left(\mathrm{VO}_{2} \max \right)\end{array}$} \\
\hline $\begin{array}{l}\text { Upper } 75 \% \\
\text { Lower } 25 \%\end{array}$ & $\begin{array}{l}1.00 \\
1.79\end{array}$ & $0.99-3.23$ & 0.054 \\
\hline \multicolumn{4}{|l|}{ Self-perceived health } \\
\hline $\begin{array}{l}\text { Third tertile } \\
\text { Second tertile } \\
\text { First tertile }\end{array}$ & $\begin{array}{l}1.00 \\
2.45 \\
3.86\end{array}$ & $\begin{array}{l}1.30-4.64 \\
1.74-8.57\end{array}$ & $\begin{array}{l}0.006 \\
0.001\end{array}$ \\
\hline
\end{tabular}

Table 3. Age-adjusted associations of health behavior with incidence of disability retirement.

\begin{tabular}{lccc}
\hline Variable & $\begin{array}{c}\text { Adjusted } \\
\text { odds ratio }\end{array}$ & $\begin{array}{c}95 \% \\
\text { confidence } \\
\text { interval }\end{array}$ & P-value \\
& & & \\
\hline Smoking & & & \\
Never & 1.00 & & 0.006 \\
Former & 2.91 & $1.37-6.22$ & 0.055 \\
Current & 2.20 & $0.99-4.90$ & \\
Alcohol & & & \\
Drinkers & & & \\
First quartile & 1.00 & & \\
Second quartile & 0.72 & $0.36-1.46$ & 0.361 \\
Third quartile & 0.64 & $0.31-1.33$ & 0.231 \\
Fourth quartile & 1.03 & $0.53-2.00$ & 0.939 \\
Abstainers (refers to all others) & 1.19 & $0.56-2.51$ & 0.651 \\
Leisure-time physical activity & & & \\
$\quad$ First quartile (shortest duration) & 1.00 & & \\
$\quad$ Second quartile & 1.02 & $0.51-2.06$ & 0.953 \\
Third quartile & 0.74 & $0.35-1.57$ & 0.443 \\
Fourth quartile & 1.01 & $0.50-2.03$ & 0.980 \\
\hline
\end{tabular}

0.78 ). As the number of working family members increased, the odds ratio for disability retirement increased from $3.42(95 \% \mathrm{Cl} 0.98-11.99)$ for 1 member to 5.04 (95\% CI $0.73-34.74$ ) for 4 or more family members working, compared with none.

\section{Disease status}

Table 2 presents age-adjusted associations between prevalent diseases, low cardiorespiratory fitness, and selfperceived health and disability retirement. Serious injury in the past (OR 2.73, 95\% CI 1.45-5.15), osteoarthritis (OR 2.19, 95\% CI 1.24-3.86), and headache (OR 2.09,

Table 4. Age-adjusted associations of physical and organizational work conditions with the incidence of disability retirement.

\begin{tabular}{|c|c|c|c|}
\hline Variable & $\begin{array}{l}\text { Adjusted } \\
\text { odds ratio }\end{array}$ & $\begin{array}{l}95 \% \\
\text { confidence } \\
\text { interval }\end{array}$ & P-value \\
\hline \multicolumn{4}{|l|}{ Physical work } \\
\hline $\begin{array}{l}\text { Light } \\
\text { Moderately active } \\
\text { Heavy or very heavy }\end{array}$ & $\begin{array}{l}1.00 \\
2.14 \\
3.27\end{array}$ & $\begin{array}{l}1.09-4.20 \\
1.61-6.63\end{array}$ & $\begin{array}{l}0.028 \\
0.001\end{array}$ \\
\hline \multicolumn{4}{|l|}{ Repetitive strain index } \\
\hline $\begin{array}{l}\text { First tertile } \\
\text { Second tertile } \\
\text { Third tertile }\end{array}$ & $\begin{array}{l}1.00 \\
1.43 \\
2.08\end{array}$ & $\begin{array}{l}0.71-2.87 \\
1.17-3.70\end{array}$ & $\begin{array}{l}0.316 \\
0.013\end{array}$ \\
\hline \multicolumn{4}{|l|}{ Work in uncomfortable position ${ }^{a}$} \\
\hline $\begin{array}{l}\text { Very little } \\
\text { Some } \\
\text { Much }\end{array}$ & $\begin{array}{l}1.00 \\
2.12 \\
2.64\end{array}$ & $\begin{array}{l}1.03-4.34 \\
1.38-5.06\end{array}$ & $\begin{array}{l}0.041 \\
0.003\end{array}$ \\
\hline \multicolumn{4}{|l|}{ Crouching } \\
\hline $\begin{array}{l}\text { None } \\
\text { Some } \\
\text { Much }\end{array}$ & $\begin{array}{l}1.00 \\
1.78 \\
2.45\end{array}$ & $\begin{array}{l}0.94-3.34 \\
1.08-5.52\end{array}$ & $\begin{array}{l}0.075 \\
0.033\end{array}$ \\
\hline \multicolumn{4}{|l|}{ Noise exposure } \\
\hline $\begin{array}{l}\text { No } \\
\text { Yes }\end{array}$ & $\begin{array}{l}1.00 \\
2.83\end{array}$ & $1.44-5.56$ & 0.003 \\
\hline \multicolumn{4}{|l|}{ Weekly workhours } \\
\hline $\begin{array}{l}<40 \text { hours } \\
40-44 \text { hours } \\
45-59 \text { hours } \\
\geq 60 \text { hours }\end{array}$ & $\begin{array}{l}1.00 \\
1.56 \\
2.04 \\
2.75\end{array}$ & $\begin{array}{l}0.70-3.51 \\
0.82-5.05 \\
1.11-6.81\end{array}$ & $\begin{array}{l}0.278 \\
0.124 \\
0.029\end{array}$ \\
\hline \multicolumn{4}{|l|}{ Overwork index } \\
\hline $\begin{array}{l}\text { Lower } 50 \% \\
\text { Upper } 50 \%\end{array}$ & $\begin{array}{l}1.00 \\
1.50\end{array}$ & $0.82-2.73$ & 0.188 \\
\hline \multicolumn{4}{|l|}{ Type of shift } \\
\hline $\begin{array}{l}\text { No shift work } \\
\text { Regular shift } \\
\text { Irregular shift }\end{array}$ & $\begin{array}{l}1.00 \\
0.29 \\
1.37\end{array}$ & $\begin{array}{l}0.07-1.24 \\
0.62-3.04\end{array}$ & $\begin{array}{l}0.095 \\
0.433\end{array}$ \\
\hline \multicolumn{4}{|l|}{ Piece work } \\
\hline $\begin{array}{l}\text { No } \\
\text { Yes }\end{array}$ & $\begin{array}{l}1.00 \\
1.87\end{array}$ & $0.87-4.03$ & 0.111 \\
\hline \multicolumn{4}{|l|}{ Deadlines } \\
\hline $\begin{array}{l}\text { Weekly or less often } \\
\text { Daily or more often }\end{array}$ & $\begin{array}{l}1.00 \\
1.52\end{array}$ & $0.90-2.58$ & 0.121 \\
\hline \multicolumn{4}{|c|}{$\begin{array}{l}\text { Opportunities for making contact } \\
\text { with fellow workers }\end{array}$} \\
\hline $\begin{array}{l}\text { Easy } \\
\text { Phone only } \\
\text { View or shout only } \\
\text { None or no fellow workers }\end{array}$ & $\begin{array}{l}1.00 \\
2.69 \\
3.76 \\
1.65\end{array}$ & $\begin{array}{l}0.75-9.63 \\
0.97-14.56 \\
0.55-4.90\end{array}$ & $\begin{array}{l}0.128 \\
0.056 \\
0.371\end{array}$ \\
\hline
\end{tabular}

a This variable was also an item of the repetitive strain index. 
95\% CI 1.12-3.89) were strongly associated with disability retirement. Rheumatoid arthritis, back problems, and diseases of the urinary tract also predicted disability retirement, but, due to the small numbers, the associations were not statistically significant. Symptomatic coronary heart disease was not associated with disability retirement, while asymptomatic coronary heart disease showed an inverse association. Two general health indicators, 1 objective (ie, $\dot{\mathrm{V}}_{2} \max$ ) and 1 subjective (ie, self-perceived health) were both strongly associated with disability retirement.

\section{Health behavior}

Table 3 summarizes the findings from the age-adjusted logistic regression models for health-related behavior. Elevated risks of disability retirement were found for former smokers (OR 2.91, 95\% CI 1.37-6.22). There was a graded, though nonsignificant, relationship between levels of current smoking and disability retirement. Neither alcohol consumption nor duration of leisure-time physical activity was related to disability retirement.

\section{Workplace factors}

Table 4 presents the age-adjusted associations between physical and organizational work conditions and the incidence of disability retirement. There was a graded positive relationship between the heaviness of work and disability retirement with an odds ratio of 3.27 (95\% CI 1.61-6.63) for men performing heavy or very heavy work. The reporting of working much in uncomfortable positions was associated with elevated risk (OR 2.64, $95 \%$ CI 1.38-5.06), as was the reporting of work involving continuous or repetitive straining of the muscles (OR 2.08, 95\% CI 1.17-3.70). Noise exposure was a strong predictor (OR 2.83, 95\% CI 1.44-5.56). The risk of disability retirement increased with the number of hours worked per week, and there was a tendency towards an increased risk with signs of overwork. Frequent deadlines at work and piece-work also tended to be associated with higher risks (OR 1.52, 95\% CI 0.90-2.58 and OR $1.87,95 \%$ CI $0.87-4.03$, respectively). The amount of standing, sitting, or walking did not predict disability retirement (data not shown).

Table 5 presents workplace factors which reflect the work environment in terms of perceived physical and mental job strain, job dissatisfaction, and support at work. Physically strenuous work was associated with an increased risk of disability retirement (OR $3.80,95 \% \mathrm{CI}$ 1.63 - 8.84 for much strain). Similar patterns of graded associations were observed for musculoskeletal strain, particularly of the back and neck, and cardiorespiratory strain. The experience of more than average mental strain or stress from strenuous work was associated with an elevated risk of disability retirement (OR 3.11, 95\% CI
1.62-5.99), as was the psychological strain index. Tiredness after work was related with disability retirement (OR 2.15, 95\% CI 1.02-4.54). Men in the upper quintile of job dissatisfaction showed an increased risk (OR $1.81,95 \%$ CI 1.04-3.16). The highest tertile of supervisor support tended to reduce the risk of disability retirement by a factor of 2 (OR 0.50, 95\% CI $0.24-1.05$ ). Co-worker support similarly tended to reduce the risk of disability retirement.

Table 5. Age-adjusted associations of job strain, dissatisfaction, and support at work with the incidence of disability retirement.

\begin{tabular}{|c|c|c|c|}
\hline Variable & $\begin{array}{l}\text { Adjusted } \\
\text { odds ratio }\end{array}$ & $\begin{array}{l}95 \% \\
\text { confidence } \\
\text { interval }\end{array}$ & P-value \\
\hline \multicolumn{4}{|l|}{ Physical strain } \\
\hline $\begin{array}{l}\text { Light } \\
\text { Average } \\
\text { Much }\end{array}$ & $\begin{array}{l}1.00 \\
1.73 \\
3.80\end{array}$ & $\begin{array}{l}0.73-4.12 \\
1.63-8.84\end{array}$ & $\begin{array}{l}0.216 \\
0.002\end{array}$ \\
\hline \multicolumn{4}{|c|}{ Musculoskeletal strain index } \\
\hline $\begin{array}{l}\text { First quartile } \\
\text { Second quartile } \\
\text { Third quartile } \\
\text { Fourth quartile }\end{array}$ & $\begin{array}{l}1.00 \\
1.81 \\
2.00 \\
2.96\end{array}$ & $\begin{array}{l}0.77-4.27 \\
0.83-4.82 \\
1.29-6.78\end{array}$ & $\begin{array}{l}0.177 \\
0.122 \\
0.011\end{array}$ \\
\hline \multicolumn{4}{|l|}{ Back strain $^{a}$} \\
\hline $\begin{array}{l}\text { Little } \\
\text { Moderate } \\
\text { Much }\end{array}$ & $\begin{array}{l}1.00 \\
1.53 \\
2.49\end{array}$ & $\begin{array}{l}0.66-3.53 \\
1.22-5.07\end{array}$ & $\begin{array}{l}0.322 \\
0.012\end{array}$ \\
\hline \multicolumn{4}{|l|}{ Neck strain ${ }^{\mathrm{a}}$} \\
\hline $\begin{array}{l}\text { Little } \\
\text { Moderate } \\
\text { Much }\end{array}$ & $\begin{array}{l}1.00 \\
1.08 \\
1.86\end{array}$ & $\begin{array}{l}0.47-2.48 \\
0.95-3.62\end{array}$ & $\begin{array}{l}0.859 \\
0.069\end{array}$ \\
\hline \multicolumn{4}{|c|}{ Cardiorespiratory strain index } \\
\hline $\begin{array}{l}\text { First quartile } \\
\text { Second quartile } \\
\text { Third quartile } \\
\text { Fourth quartile }\end{array}$ & $\begin{array}{l}1.00 \\
1.27 \\
1.47 \\
2.18\end{array}$ & $\begin{array}{l}0.59-2.74 \\
0.67-3.21 \\
1.00-4.77\end{array}$ & $\begin{array}{l}0.547 \\
0.338 \\
0.051\end{array}$ \\
\hline \multicolumn{4}{|l|}{ Psychological strain index } \\
\hline $\begin{array}{l}\text { None } \\
\text { Average } \\
\text { Much }\end{array}$ & $\begin{array}{l}1.00 \\
1.28 \\
2.25\end{array}$ & $\begin{array}{l}0.70-2.35 \\
1.10-4.59\end{array}$ & $\begin{array}{l}0.428 \\
0.026\end{array}$ \\
\hline \multicolumn{4}{|c|}{ Mental strain from strenuous work } \\
\hline $\begin{array}{l}\text { None } \\
\text { Average } \\
\text { Much }\end{array}$ & $\begin{array}{l}1.00 \\
2.42 \\
3.11\end{array}$ & $\begin{array}{l}1.23-4.78 \\
1.62-5.99\end{array}$ & $\begin{array}{l}0.011 \\
0.001\end{array}$ \\
\hline \multicolumn{4}{|l|}{ Time pressure } \\
\hline $\begin{array}{l}\text { First tertile } \\
\text { Second tertile } \\
\text { Third tertile }\end{array}$ & $\begin{array}{l}1.00 \\
1.26 \\
2.03\end{array}$ & $\begin{array}{l}0.62-2.59 \\
1.00-4.11\end{array}$ & $\begin{array}{l}0.522 \\
0.051\end{array}$ \\
\hline \multicolumn{4}{|l|}{ Tiredness after work } \\
\hline $\begin{array}{l}\text { Lively } \\
\text { As often lively as tired } \\
\text { Tired }\end{array}$ & $\begin{array}{l}1.00 \\
2.42 \\
2.15\end{array}$ & $\begin{array}{l}1.15-5.11 \\
1.02-4.54\end{array}$ & $\begin{array}{l}0.020 \\
0.044\end{array}$ \\
\hline \multicolumn{4}{|l|}{ Job dissatisfaction } \\
\hline $\begin{array}{l}\text { Lower } 80 \% \\
\text { Upper } 20 \%\end{array}$ & $\begin{array}{l}1.00 \\
1.81\end{array}$ & $1.04-3.16$ & 0.036 \\
\hline \multicolumn{4}{|l|}{ Co-worker support } \\
\hline $\begin{array}{l}\text { First tertile } \\
\text { Second tertile } \\
\text { Third tertile }\end{array}$ & $\begin{array}{l}1.00 \\
0.60 \\
0.64\end{array}$ & $\begin{array}{l}0.34-1.06 \\
0.30-1.35\end{array}$ & $\begin{array}{l}0.076 \\
0.242\end{array}$ \\
\hline \multicolumn{4}{|l|}{ Supervisor support } \\
\hline $\begin{array}{l}\text { First tertile } \\
\text { Second tertile } \\
\text { Third tertile }\end{array}$ & $\begin{array}{l}1.00 \\
1.28 \\
0.50\end{array}$ & $\begin{array}{l}0.71-2.30 \\
0.24-1.05\end{array}$ & $\begin{array}{l}0.406 \\
0.062\end{array}$ \\
\hline
\end{tabular}

a This variable was also an item of the musculoskeletal strain index. 
Work conditions adjusted for economic factors, prevalent diseases and health behavior

Table 6 displays the age-adjusted associations between each work condition and disability retirement (column 1) with incremental adjustments for economic factors (column 2), prevalent diseases (column 3), and health behavior (column 4).

Risk estimates for physical and organizational work conditions remained virtually unchanged after control for age and economic factors. For example, heavy work,

Table 6. Associations of physical and organizational work conditions with the incidence of disability retirement by incremental adjustments for age, economic factors, prevalent diseases, and health behavior.

\begin{tabular}{|c|c|c|c|c|}
\hline \multirow[t]{2}{*}{ Variable } & \multicolumn{4}{|c|}{ Adjusted odds ratios ${ }^{a}$} \\
\hline & Age & $\begin{array}{l}\text { Age plus } \\
\text { economic } \\
\text { factors }\end{array}$ & $\begin{array}{c}\text { Age plus } \\
\text { economic } \\
\text { factors plus } \\
\text { prevalent } \\
\text { diseases }^{b}\end{array}$ & $\begin{array}{c}\text { Age plus } \\
\text { economic } \\
\text { factors }{ }^{\mathrm{a}} \text { plus } \\
\text { prevalent } \\
\text { diseases }^{\mathrm{b}} \text { plus } \\
\text { health } \\
\text { behavior }\end{array}$ \\
\hline \multicolumn{5}{|l|}{ Physical work } \\
\hline $\begin{array}{l}\text { Light } \\
\text { Moderately active } \\
\text { Heavy or very heavy }\end{array}$ & $\begin{array}{l}1.00 \\
2,14^{* *} \\
3.27^{\star *}\end{array}$ & $\begin{array}{l}2.29^{*} \\
3.63^{* *}\end{array}$ & $\begin{array}{l}2.12^{\star *} \\
3.46^{\star *}\end{array}$ & $\begin{array}{l}2.13^{\star \star} \\
3.56^{* \star}\end{array}$ \\
\hline \multicolumn{5}{|l|}{ Repetitive strain index } \\
\hline $\begin{array}{l}\text { First tertile } \\
\text { Second tertile } \\
\text { Third tertile }\end{array}$ & $\begin{array}{l}1.00 \\
1.43 \\
2.08^{* *}\end{array}$ & $\begin{array}{l}1.39 \\
2.01^{* *}\end{array}$ & $\begin{array}{l}1.24 \\
1.62\end{array}$ & $\begin{array}{l}1.19 \\
1.61\end{array}$ \\
\hline \multicolumn{5}{|l|}{ Work in uncomfortable position ${ }^{d}$} \\
\hline $\begin{array}{l}\text { Very little } \\
\text { Some } \\
\text { Much }\end{array}$ & $\begin{array}{l}1.00 \\
2.12^{* *} \\
2.64^{* *}\end{array}$ & $\begin{array}{l}2.03^{*} \\
2.60^{* *}\end{array}$ & $\begin{array}{l}1.83 \\
2.02^{*}\end{array}$ & $\begin{array}{l}1.95^{*} \\
1.98^{*}\end{array}$ \\
\hline \multicolumn{5}{|l|}{ Crouching } \\
\hline $\begin{array}{l}\text { None } \\
\text { Some } \\
\text { Much }\end{array}$ & $\begin{array}{l}1.00 \\
1.78^{*} \\
2.45^{\star \star}\end{array}$ & $\begin{array}{l}1.78^{*} \\
2.32^{*}\end{array}$ & $\begin{array}{l}1.88^{*} \\
1.63\end{array}$ & $\begin{array}{l}2.04 \\
1.72\end{array}$ \\
\hline \multicolumn{5}{|l|}{ Noise exposure } \\
\hline $\begin{array}{l}\text { No } \\
\text { Yes }\end{array}$ & $\begin{array}{l}1.00 \\
2.83^{* \star}\end{array}$ & $2.85^{\star \star}$ & $2.54^{\star \star}$ & $2.46^{* \star}$ \\
\hline \multicolumn{5}{|l|}{ Weekly work hours } \\
\hline $\begin{array}{l}<40 \text { hours } \\
40-44 \text { hours } \\
45-59 \text { hours } \\
\geq 60 \text { hours }\end{array}$ & $\begin{array}{l}1.00 \\
1.56 \\
2.04 \\
2.75^{\star *}\end{array}$ & $\begin{array}{l}1.42 \\
1.99 \\
2.87^{* *}\end{array}$ & $\begin{array}{l}1.28 \\
1.59 \\
2.15\end{array}$ & $\begin{array}{l}1.26 \\
1.49 \\
2.49^{*}\end{array}$ \\
\hline \multicolumn{5}{|l|}{ Overwork index } \\
\hline $\begin{array}{l}\text { Lower } 50 \% \\
\text { Upper } 50 \%\end{array}$ & $\begin{array}{l}1.00 \\
1.50\end{array}$ & 1.51 & 1.31 & 1.31 \\
\hline \multicolumn{5}{|l|}{ Type of shift } \\
\hline $\begin{array}{l}\text { No shift work } \\
\text { Regular shift } \\
\text { Irregular shift }\end{array}$ & $\begin{array}{l}1.00 \\
0.29^{*} \\
1.37\end{array}$ & $\begin{array}{l}0.33 \\
1.38\end{array}$ & $\begin{array}{l}0.26^{\star} \\
1.14\end{array}$ & $\begin{array}{l}0.24^{*} \\
0.98\end{array}$ \\
\hline \multicolumn{5}{|l|}{ Piece work } \\
\hline $\begin{array}{l}\text { No } \\
\text { Yes }\end{array}$ & $\begin{array}{l}1.00 \\
1.87\end{array}$ & 1.61 & 1.25 & 1.22 \\
\hline \multicolumn{5}{|l|}{ Deadliness } \\
\hline $\begin{array}{l}\text { Weekly or less often } \\
\text { Daily or more often }\end{array}$ & $\begin{array}{l}1.00 \\
1.52\end{array}$ & $1.59^{*}$ & 1.25 & 1.22 \\
\hline \multicolumn{5}{|c|}{$\begin{array}{l}\text { Opportunities for making contact } \\
\text { with fellow workers }\end{array}$} \\
\hline $\begin{array}{l}\text { Easy } \\
\text { Phone only } \\
\text { View or shout only } \\
\text { None or no fellow workers }\end{array}$ & $\begin{array}{l}1.00 \\
2.69 \\
3.76^{\star} \\
1.65\end{array}$ & $\begin{array}{l}2.65 \\
4.44^{* *} \\
1.91\end{array}$ & $\begin{array}{l}2.21 \\
3.63 \\
2.24\end{array}$ & $\begin{array}{l}2.44 \\
3.84 \\
2.84\end{array}$ \\
\hline
\end{tabular}

a The economic factors included income, unemployment in past 5 years, and number of family members working.

b The prevalent diseases included serious injury in the past, osteoarthritis, rheumatoid arthritis, back problems, headache, depression, obesity, urinary tract disease, digestive tract disease, respiratory disease, symptomatic and asymptomatic coronary heart disease, and cardirespiratory fitness (maximal oxygen consumption).

c Health behavior included smoking, alcohol, and leisure-time physical activity.

- This variable was also an item of the repetitive strain index.

${ }^{*} P<0.10,{ }^{* *} P<0.05$. 
after additional adjustment for economic factors, showed an odds ratio of $3.63(95 \% \mathrm{CI} 1.66-7.92)$ compared with an odds ratio of $3.27(95 \%$ CI $1.61-6.63)$ in the simple age-adjusted model.

Adjustment for disease status was obtained by including indicator variables for each diagnostic group that was importantly associated with disability retirement and for being in the lowest quartile of cardiorespiratory fitness. Although the associations were reduced to some degree (eg, the odds ratio decreased from 3.63 to 3.46 in the case of heavy work) most of the work conditions showed independent effects on disability retirement even after adjustment for age, economic factors, prevalent diseases, and cardiorespiratory fitness.

The last column in table 6 shows risk estimates for work conditions after additional adjustment for healthrelated behavior factors (ie, smoking, alcohol consumption, and leisure-time physical activity). Adjustment for behavioral factors had little effect on the risk estimates for work conditions.

\section{Job strain, dissatisfaction and support at work, adjusted for economic factors, diagnosed diseases and health behavior}

Table 7 displays the associations for job strain, job dissatisfaction, and social support at work after incremental adjustments for economic factors, prevalent diseases, and health behavior. Adjustment for economic variables did not markedly change the risk estimates. Additional adjustment for disease status reduced most risk estimates with the exception of the psychological strain index, social support, and job dissatisfaction. Further adjustment for health behavior did not notably alter the associations.

\section{Discussion}

This is the first prospective population-based study of predictors of disability retirement with the ability to examine workplace, socioeconomic, behavioral, and healthrelated factors simultaneously. Socioeconomic factors, physical and psychosocial characteristics of the work environment, prevalent diagnosed disease, self-perceived health, and smoking behavior all predicted disability retirement in this population-based sample of middle-aged Finnish men. The associations between workplace factors and disability retirement largely persisted after control for economic variables, disease status, and health behavior. Specifically, we found that job characteristics such as heavy work, working in uncomfortable positions, working in isolation from fellow workers, noise exposure, and long workhours were associated with increased risk of disability retirement. Reports of physical, musculoskeletal, cardiovascular, and mental job strain, tiredness after work, and job dissatisfaction were also related to disability retirement. Social support at work, both from supervisors and co-workers tended to reduce the risk of disability retirement. Control for disease status reduced the magnitude of the risk associated with physical and organizational work conditions and physical job strain but not the risk associated with psychological strain, job dissatisfaction, or social support.

These findings are significant in several ways. First, the results were obtained from a population-based sample, avoiding the selection biases that may limit interpretability of many industry-based studies. Second, the prospective design established a clear temporal relationship between predictors and outcome necessary for causal interpretation. Third, the data allowed for a differentiation between questions which measure more objective characteristics of the work environment (eg, hours, crouching) versus more subjective characteristics of the work environment (eg, mental strain, social support). Interestingly, corresponding domains tended to produce similar results via both types of question. For example, both frequency of awkward work positions like crouching (an objective job characteristic) and perceived musculoskeletal strain (a more subjective job characteristic) showed equally strong relationships with disability retirement. Of course, all factors were based on self-reports, and it was not possible to validate these reports independently against direct observation. Other studies suggest that the correlation between self-reports and direct observation is not always satisfactory and differs by the type of work condition examined (2024). The potential for bias has been shown to be lowest for prospective studies (25). Fourth, the assessment of prevalent diseases was based on an extensive and elaborate medical history and examination including a wide range of laboratory tests performed over a 2 -day period. Fifth, the study also controlled for health behavior factors that were assessed by standardized questionnaires that have been predictive of several health outcomes in this population, including myocardial infarction and mortality.

In this study, alcohol consumption and leisure-time physical activity were not related to disability retirement, but former and current smoking were independently related to disability retirement even after disease status and socioeconomic variables were taken into account. However, smoking habits did not reduce the risk estimates associated with any job factor. As a consequence it appears that worksite health promotion focusing on smoking cessation programs and behavioral change in terms of reduced alcohol consumption or increased leisuretime physical activity may not substitute for actual job redesign in lowering the incidence of disability retirement.

Scand J Work Environ Health 1997, vol 23, no 6 
Table 7. Associations of job strain, dissatisfaction, and support at work with the incidence of disability retirement by incremental adjustments for age, economic factors, prevalent diseases, and health behavior.

\begin{tabular}{|c|c|c|c|c|}
\hline \multirow[t]{2}{*}{ Variable } & \multicolumn{4}{|c|}{ Adjusted odds ratios ${ }^{\mathrm{a}}$} \\
\hline & Age & $\begin{array}{l}\text { Age plus } \\
\text { economic } \\
\text { factors }\end{array}$ & $\begin{array}{l}\text { Age plus } \\
\text { economic } \\
\text { factorsa plus } \\
\text { prevalent } \\
\text { diseases }^{b}\end{array}$ & $\begin{array}{c}\text { Age plus } \\
\text { economic } \\
\text { factorsa plus } \\
\text { prevalent } \\
\text { diseases plus } \\
\text { health } \\
\text { behavior }\end{array}$ \\
\hline \multicolumn{5}{|l|}{ Physical strain } \\
\hline $\begin{array}{l}\text { Light } \\
\text { Average } \\
\text { Much }\end{array}$ & $\begin{array}{l}1.00 \\
1.73 \\
3.80\end{array}$ & $\begin{array}{l}1.75 \\
4.05^{\star *}\end{array}$ & $\begin{array}{l}1.77 \\
3.29^{* \star}\end{array}$ & $\begin{array}{l}1.91 \\
3.43^{\text {* }}\end{array}$ \\
\hline \multicolumn{5}{|c|}{ Musculoskeletal strain index } \\
\hline $\begin{array}{l}\text { First quartile } \\
\text { Second quartile } \\
\text { Third quartile } \\
\text { Fourth quartile }\end{array}$ & $\begin{array}{l}1.00 \\
1.81 \\
2.00 \\
2.96\end{array}$ & $\begin{array}{l}1.77 \\
2.05 \\
2.97^{\star *}\end{array}$ & $\begin{array}{l}1.65 \\
1.75 \\
1.86\end{array}$ & $\begin{array}{l}1.79 \\
1.87 \\
1.91\end{array}$ \\
\hline \multicolumn{5}{|l|}{ Back strain ${ }^{\circ}$} \\
\hline $\begin{array}{l}\text { Little } \\
\text { Moderate } \\
\text { Much }\end{array}$ & $\begin{array}{l}1.00 \\
1.53 \\
2.49\end{array}$ & $\begin{array}{l}1.51 \\
2.43^{* *}\end{array}$ & $\begin{array}{l}1.26 \\
1.83\end{array}$ & $\begin{array}{l}1.30 \\
1.80\end{array}$ \\
\hline \multicolumn{5}{|l|}{ Neck straind } \\
\hline $\begin{array}{l}\text { Little } \\
\text { Moderate } \\
\text { Much }\end{array}$ & $\begin{array}{l}1.08 \\
1.86\end{array}$ & $\begin{array}{l}1.08 \\
1.81^{*}\end{array}$ & $\begin{array}{l}0.92 \\
1.39\end{array}$ & $\begin{array}{l}1.02 \\
1.38\end{array}$ \\
\hline \multicolumn{5}{|c|}{ Cardiorespiratory strain index } \\
\hline $\begin{array}{l}\text { First quartile } \\
\text { Second quartile } \\
\text { Third quartile } \\
\text { Fourth quartile }\end{array}$ & $\begin{array}{l}1.00 \\
1.27 \\
1.47 \\
2.18\end{array}$ & $\begin{array}{l}1.16 \\
1.36 \\
2.16\end{array}$ & $\begin{array}{l}1.10 \\
1.09 \\
1.46\end{array}$ & $\begin{array}{l}1.14 \\
1.14 \\
1.44\end{array}$ \\
\hline \multicolumn{5}{|l|}{ Psychological strain index } \\
\hline $\begin{array}{l}\text { None } \\
\text { Average } \\
\text { Much }\end{array}$ & $\begin{array}{l}1.00 \\
1.28 \\
2.25\end{array}$ & $\begin{array}{l}1.27 \\
2.13^{*}\end{array}$ & $\begin{array}{l}1.12 \\
2.05^{*}\end{array}$ & $\begin{array}{l}1.14 \\
1.88\end{array}$ \\
\hline \multicolumn{5}{|c|}{ Mental strain from strenuous work } \\
\hline $\begin{array}{l}\text { None } \\
\text { Average } \\
\text { Much }\end{array}$ & $\begin{array}{l}1.00 \\
2.42 \\
3.11\end{array}$ & $\begin{array}{l}2.35^{\star *} \\
3.24^{* *}\end{array}$ & $\begin{array}{l}2.10^{* *} \\
2.34^{* *}\end{array}$ & $\begin{array}{l}2.18^{* *} \\
2.45^{* *}\end{array}$ \\
\hline \multicolumn{5}{|l|}{ Time pressure } \\
\hline $\begin{array}{l}\text { First tertile } \\
\text { Second tertile } \\
\text { Third tertile }\end{array}$ & $\begin{array}{l}1.00 \\
1.26 \\
2.03\end{array}$ & $\begin{array}{l}1.27 \\
2.18^{\star}\end{array}$ & $\begin{array}{l}1.19 \\
1.65\end{array}$ & $\begin{array}{l}1.21 \\
1.62\end{array}$ \\
\hline \multicolumn{5}{|l|}{ Tiredness after work } \\
\hline $\begin{array}{l}\text { Lively } \\
\text { As often lively as tired } \\
\text { Tired }\end{array}$ & $\begin{array}{l}1.00 \\
2.42 \\
2.15\end{array}$ & $\begin{array}{l}2.53^{\star *} \\
2.22^{* \star}\end{array}$ & $\begin{array}{l}2.00^{*} \\
2.10^{*}\end{array}$ & $\begin{array}{l}2.00 \\
2.17^{\star}\end{array}$ \\
\hline \multicolumn{5}{|l|}{ Job dissatisfaction } \\
\hline $\begin{array}{l}\text { Lower } 80 \% \\
\text { Upper } 20 \%\end{array}$ & $\begin{array}{l}1.00 \\
1.81^{* *}\end{array}$ & $1.80^{\star \star}$ & $2.23^{\star *}$ & $2.05^{* *}$ \\
\hline \multicolumn{5}{|l|}{ Co-worker support } \\
\hline $\begin{array}{l}\text { First tertile } \\
\text { Second tertile } \\
\text { Third tertile }\end{array}$ & $\begin{array}{l}1.00 \\
0.60 \\
0.64\end{array}$ & $\begin{array}{l}0.60^{*} \\
0.69\end{array}$ & $\begin{array}{l}0.66 \\
0.69\end{array}$ & $\begin{array}{l}0.55^{*} \\
0.61\end{array}$ \\
\hline \multicolumn{5}{|l|}{ Supervisor support } \\
\hline $\begin{array}{l}\text { First tertile } \\
\text { Second tertile } \\
\text { Third tertile }\end{array}$ & $\begin{array}{l}1.00 \\
1.28 \\
0.50\end{array}$ & $\begin{array}{l}1.42 \\
0.53\end{array}$ & $\begin{array}{l}1.46 \\
0.52^{\star}\end{array}$ & $\begin{array}{l}1.50 \\
0.44^{* *}\end{array}$ \\
\hline
\end{tabular}

a The economic factors included income, unemployment in past 5 years, and number of family members working.

- Prevalent diseases included serious injury in the past, osteoarthritis, rheumatoid arthritis, back problems, headaches, depression, obesity, urinary tract disease, digestive tract disease, respiratory disease, symptomatic and asymptomatic coronary heart disease, and cardiorespiratory fitness (maximal oxygen consumption).

Gealth behavior included smoking, alcohol, and leisure-time physical activity.

This variable was also an item of the musculoskeletal strain index.

$* P<0.10,{ }^{* *} P<0.05$. 
Several authors have discussed the possible impact of economic incentives on decisions to take retirement (26-30). Some findings of this study support the notion that economic considerations might play a role in this process. The overall financial stability of the family measured in number of family members working or unemployed seemed to influence the incidence of disability retirement. Interestingly, the level of personal or household income per se did not predict disability retirement in this study. Some economists have proposed that medically diagnosed disease is a result of a rational choice element in employee behavior expressed in the act of seeking medical evaluation. The argument is that such choice is influenced by economic incentives for retirement depending on the expectations regarding retirement income. It is important to note that, in contrast to the situation in most retrospective studies, in this study the medical evaluation was neither sought by the study participants nor the social security administration, and the evaluation of disease status was based on the medical examination by researchers rather than on retirement records. Therefore the argument of choice with regard to observed disease effects seems not to be applicable. To examine the second part of the argument (ie, the question of whether income after retirement establishes an incentive for disability retirement), we compared the income change in incident cases of disability retirement from the beginning of the study to the time of the follow-up with the corresponding changes for workers not retiring during this period. The mean change for the disability retirees was a reduction of FIM 4736 in yearly income compared with an increase of FIM 19696 for the working study participants within the follow-up period, leaving the disability retirees with $65 \%$ of the median income of their working peers compared with $96 \%$ before retirement. Clearly, these data do not support an "economic inducement theory" of disability retirement based solely on expected individual retirement income.

The study has several limitations. Disease status and other covariates may have changed during the follow-up period. Because of the small number of events, it was not feasable to analyze multiple workplace factors concurrently while adjusting for major socioeconomic and health status variables. The small number of events made it also impossible to stratify results by economic sector or occupational group. To identify the most pressing needs for job redesign for each industry, it would be very informative to conduct similar analyses in a larger sample, with stratification by economic sector or occupational group. Finally, simultaneous measurement of disease status, work conditions, and economic factors did not allow for temporal relationships to be established among covariates, and it precluded meaningful pathway analyses in this study.
Although it is not surprising that disease status is a predictor of disability retirement, including diseases as independent covariates in multivariate analyses may obscure the fact that some diseases may be, in part, the result of work conditions (31). The result would be an underestimation of the effect of workplace factors. We were able to examine this question in our sample for the case of osteoarthritis. Preliminary analyses showed that some of the workplace factors that predicted disability retirement also predicted the incidence of osteoarthritis. Therefore, prevalent diseases should probably not be considered as confounders but, instead, as part of the causal pathway.

Despite these limitations, and in light of the paucity of published data on the determinants of disability retirement, the strong associations found in this study for many workplace factors suggest a great potential for preventing disability retirement through job redesign. The results suggest that redesign of the physical and organizational work environment, reducing heaviness of work and job strain and enhancing job satisfaction and social support at work, could be important avenues for preventing the decline of labor force participation due to disability retirement among men after 42 years of age and the resultant loss of skilled and productive workers. The results of this study link the problem of disability retirement to the problem of creating healthy and satisfying work conditions. Future investigations should identify which work conditions are of most importance for the retirement risk of different occupational groups. Such analyses would help to set priorities for specific prevention programs at the workplace.

\section{Acknowledgments}

This study was supported by grant HL44199 from the National Heart, Lung, and Blood Institute in the United States and by grants from the Academy of Finland and the Finnish Ministry of Education.

\section{References}

1. Rantanen J. Opening address. Presented at the International Scientific Symposium on Aging and Work. Haikko (Finland) in 1992.

2. Palmore EB, George LK, Fillenbaum GG. Predictors of retirement. J Gerontol 1982;37(6):733-42.

3. Parnes HS, Sommers DG. Shunning retirement: work experience of men in their seventies and early eighties. $\mathbf{J}$ Gerontol 1994;49(3):S117-24.

4. Morris JK, Cook DG, Shaper AG. Loss of employment and mortality. BMJ 1994;308(6937):1135-9.

5. Tuomi K, Järvinen E, Eskelinen L, Ilmarinen J, Klockars M. 
Effect of retirement on health and work ability among municipal employees. Scand J Work Environ Health 1991;17 suppl 1:75-81.

6. Astrand N-E, Isacsson S-O, Olhagen GO. Prediction of early retirement on the basis of a health examination: an 11-year follow-up of 264 male employees in a Swedish pulp and paper company. Scand J Work Environ Health 1988;14:110-7.

7. Mansson NO, Israelsson B. Middle-aged men before and after disability pension: health screening profile with special emphasis on alcohol consumption. Scand J Soc Med 1987;15(3): $185-9$.

8. Tuomi K, Eskelinen L, Toikkanen J, Järvinen E, Ilmarinen J, Klockars $M$. Work load and individual factors affecting work ability among aging municipal employees. Scand J Work Environ Health 1991;17 suppl 1:128-34.

9. Tuomi K, Toikkanen J, Eskelinen L, Backman A-L, Ilmarinen J, Järvinen E, et al. Mortality, disability and changes in occupation among aging municipal employees. Scand J Work Environ Health 1991;17 suppl 1:58-66.

10. Vingård E, Lars A, Evy F, Christer H. Disability pensions due to musculo-skeletal disorders among men in heavy occupations. Scand J Soc Med 1992;20(1):31-6.

11. Yelin EH. The myth of malingering: why individuals withdraw from work in the presence of illness. Milbank Q, 1986;64(4):622-49.

12. Salonen JT. Is there a continuing need for longitudinal epidemiologic research? - the Kuopio ischaemic heart disease risk factor study. Ann Clin Res 1988;20:46-50.

13. Lakka TA, Salonen JT. Physical activity and serum lipids: a cross-sectional population study in Eastern Finnish men. Am J Epidemiol 1992;136:806-18.

14. Lynch J, Kaplan GA, Salonen R, Cohen RD, Salonen JT. Socioeconomic status and carotid atherosclerosis. Circulation 1995;92:1786-92.

15. Rose GA, Blackburn H Gillum RF. Cardiovascular survey methods. Geneva: World Health Organization, 1982.

16. Green RL. The MMPI: an interpretative manual. New York (NY): The Psychological Corporation, 1980.

17. Kauhanen J, Julkunen J, Wilson T, Salonen J. Coping with inner feelings and stress: heavy alcohol use in the context of alexithymia. Behav Med 18:121-126, 1992

18. Lakka T, Venäläinen JM, Rauramaa R, Salonen R, Tuomileh- to J, Salonen JT. Relation of leisure-time physical activity and cardiorespiratory fitness to the risk of acute myocardial infarction. N Engl J Med 1994;330:1549—54.

19. SAS Institute. SAS user's guide: statistics. Cary (NC): SAS Institute, 1990.

20. Burdorf A, Laan J. Comparison of methods for the assessment of postural load on the back. Scand J Work Environ Health 1991;17(6):425-9.

21. Greiner BA, Ragland DR, Krause N, Syme SL, Fisher M. Objective measurement of occupational stress factors - an example with San Francisco urban transit operators. J Occup Health Psychol 1997;2(4):1-18

22. Rossignol M, Baetz J. Task-related risk factors for spinal injury: validation of a self-administered questionnaire on hospital employees. Ergonomics 1987;30(11):1531-40.

23. Wiktorin C, Hjelm EW, Winkel J, Koster M, Stockholm MUSIC I Study Group. Reproducibility of a questionnaire for assessment of physical load during work and leisure time. J Occup Environ Med 1996;38(2):190—201

24. Wiktorin C, Karlqvist L, Winkel J, Stockholm MUSIC I Study Group. Validity of self-reported exposures to work postures and manual materials handling. Scand J Work Environ Health 1993;19(3):208-14.

25. Burdorf A. Bias in risk estimates from variability of exposure to postural load on the back in occupational groups. Scand J Work Environ Health 1993:19(1):50-4.

26. Bound $\mathbf{J}$. The health and earnings of rejected disability insurance applicants. Am Econ Rev 1989;79(3):482-503

27. Bound $\mathrm{J}$. The health and earnings of rejected disability. insurance applicants: reply. Am Econ Rev 1991;81(5):1427-34.

28. Chirikos TN. The relationship between health and labor market status. Ann Rev Public Health 1993;14:293-312.

29. Gould R, Takala M. Pension or work - the preferences of older workers. Presented at the International Scientific Symposium on Aging and Work, Haikko (Finland) in 1992.

30. Parsons DO. The health and earnings of rejected disability insurance applicants [comment]. Am Econ Rev 1991;81(5): 1419-26.

31. Karvonen M, Mikheer MI, editors. Epidemiology of occupational health. Copenhagen: World Health Organization (WHO), Regional Office for Europe, 1986. WHO regional publications, European series, no 20.

\section{Appendix}

\section{Description of indices of workplace factors}

Repetitive strain index: ratings of strain caused by different activities at work, including continuous lifting, continuous or repeated straining of muscles, and uncomfortable work positions ( 3 items, Cronbach's alpha 0.87)

Overwork index: combines questions about the frequency of working after hours, taking work home, thinking about work after work, and taking less than regular vacation (4 items, Cronbach's alpha 0.63)
Musculoskeletal strain index: combines answers to how much strain was felt during work in different parts of the extremities, the back, and the neck and shoulders ( 8 items, Cronbach's alpha 0.91)

Cardiorespiratory strain index: questions about frequency of sweating, breathlessness, and heart palpitation at work. (3 items, cronbach's alpha 0.82) 
Psychological strain index: combines questions about excessive supervising of schedule, troublesome supervisors, co-workers, fellow workers, or customers, job responsibility, ill-defined tasks or responsibilities, risk of accidents, risk of unemployment, irregularities of the work schedule, and strenousness of work (10 items, Cronbach's alpha 0.80)

Time pressure index: combines questions about frequency of being pressed for time, stress from deadlines, and forcing oneself to go on the same speed when getting tired at work ( 3 items, Cronbach's alpha 0.42)

Job dissatisfaction index: ratings of enjoyment of work, confidence at work, and meaningfulness of work, combined with questions about willingness to go to work and general feelings about job (5 items, Cronbach's alpha $0.77)$

Received for publication: 15 April 1997 